\title{
Weld Pool Surface Fluctuations Sensing in Pulsed GMAW
}

\author{
The characteristic information of joint penetration is analyzed using \\ structured laser reflection and electrical signal sensing in GMAW-P
}

BY W. WANG, Z. WANG, S. HU, P. BAI, T. LU, AND Y. CAO

\begin{abstract}
This work addresses the sensing and monitoring of joint penetration in pulsed gas metal arc welding (GMAW-P). The issue being addressed is challenging because the joint penetration is not directly observable, and its solution lies on the successful identification of easily measurable signals that have an inherent relationship with the joint penetration. The metal transfer in gas metal arc welding and the pulsing of the current add additional complexities in the process, causing the significant fluctuation in weld pool that disqualifies state-of-the-art methods in weld pool measurement. Through critical analysis of the complex process, the authors hypothesize that the fluctuation behavior of the weld pool under pulsing current is determined by the joint penetration. To test this hypothesis, a series of experiments were designed, and the reflection from the weld pool surface during the peak current period was used to derive a measurement to quantify the fluctuation. Experimental results showed that the fluctuation of structured laser reflection during the peak current period, which was represented by the cover area of laser reflections $\left(A_{c}\right)$ in the fixed window during a peak current period, can reflect the joint penetration in GMAW-P but with a time delay. The change in arc voltage during a peak current period $(\Delta U)$ and the average voltage during a peak current period $(\bar{U})$ can both be used to characterize joint penetration in GMAW-P. The $\Delta U$ decreases before the weld gets penetrated and then increases, while $\bar{U}$ increases before the weld gets penetrated and then decreases. These two electrical variables could be fused together to control joint penetration in GMAW-P in manufacturing plants.
\end{abstract}

\section{KEYWORDS}

- Average Arc Voltage $\bullet$ Change in Arc Voltage

- Cover Area of Laser Reflections - Peak Current Period

- Joint Penetration Sensing • Weld Pool Surface Fluctuation

\section{Introduction}

Joint penetration plays an important role in determining weld quality, and its real-time sensing and control are crucial. In many demanding applications, such as steel catenary riser welding in offshore platforms or pressure vessel welding, complete joint penetration of the root pass is strictly required.

In recent years, there have been many progresses in joint penetration sensing and control in gas tungsten arc welding (GTAW), in which the process and the weld pool are stable, and the influence from the arc is relatively weak owing to low welding current. Visual sensing of joint penetration in GTAW has been a focus because weld pool images have intuitive and abundant information, which could be understood and decoded by a skilled human welder. Chen et al. developed an algorithm based on shape from shading (SFS) to process a captured image of the weld pool, and successfully reconstructed the surface shape of the weld pool in GTAW (Refs. 1, 2). A structured-light vision system was developed to measure the shape of the free weld pool surface (Ref. 3). Song and Zhang developed an effective system with the use of the laser reflection and dot-matrix pattern to sense and reconstruct the weld pool surface in GTAW to mimic the observation of the weld pool by welders (Refs. 4, 5). Zhang et al. established a real-time vision-based monitoring system to reconstruct and measure the weld pool surface in GTAW, and the average time of reconstruction was $3.22 \mathrm{~ms}$ (Ref. 6). The joint penetration can also be characterized by the boundary geometrical parameters of the weld pool extracted directly from the captured images of the weld pool (Refs. 7, 8). The weld pool oscillation frequency is another important signal to characterize the joint penetration. The oscillation frequency of the weld pool based on arc voltage was studied, and results showed that it has a strong relationship with joint penetration (Refs. 9-11). Xiao and den Ouden measured the oscillation frequency and amplitude by monitoring the arc voltage and reported that joint penetration can be in-process controlled by monitoring the oscillation fre- 


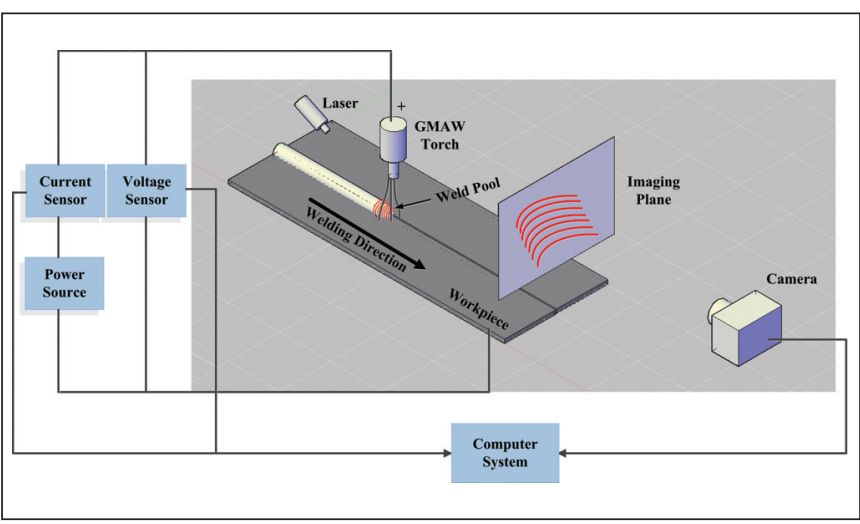

Fig. 1- Experimental setup.

quency during the welding process (Ref. 10). Wang et al. proposed a new way, using a separately excited resonance phenomenon of the weld pool, to detect real-time information of weld pool size directly from the arc voltage signal, and the realtime control of bead appearance was realized successfully in thin steel plate welding (Ref. 12). Shi et al. projected a five-line laser pattern onto the entire weld pool surface and found the relationship between the variation of reflected laser lines, which was captured by a high-speed camera during the base current period, and weld pool oscillation (Ref. 13). A laser dotmatrix sensor was used to project laser dots onto the weld pool surface in GTAW, and the brightness of captured image of laser dots reflection from the weld pool surface was detected to determine the oscillation state (i.e., a laser dot reflected from a more severely oscillating pool surface changes its reflection direction more significantly within a given small time interval, resulting in a less clear reflection image), which is correlated to the joint penetration (Ref. 14). A laser dot-matrix sensor was also used to observe the pool oscillation three dimensionally (Ref. 15). The oscillation modes were revealed and the dynamic real-time evolution process for pool oscillation under different penetration states were explored according to the shape variation of surface oscillation of the weld pool under various welding conditions of pulse current and penetration. Aside from the vision and oscillation frequency sensing above, ultrasonic (Ref. 16), x-ray (Refs. 17, 18), infrared (Ref. 19), and acoustic sensing (Ref. 20) were also studied in GTAW.

However, the joint penetration sensing and control in gas metal arc welding (GMAW) are much more challenging because of metal transfer in the process. Pulsed GMAW (GMAW-P) is even more difficult as pulse current introduces pulse arc pressure on the weld pool surface. Yan et al. established a visual sensing system to monitor the weld pool in GMAW-P and developed image processing algorithms to extract the weld pool edge from the images acquired at the end

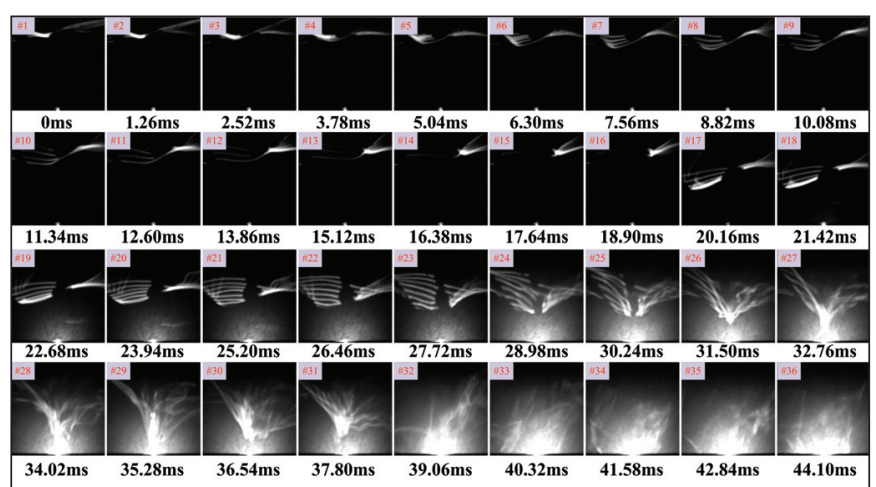

Fig. 2 - The laser reflection images at the wire feed speed (WFS) of $4.7 \mathrm{~m} / \mathrm{min}$.

of each base current period, and the characteristic information of the weld pool boundary profile was obtained in fast welding of thin plates (Refs. 21, 22). Chen et al. established a visual sensing system for monitoring weld pool variation during base current periods and realized the real-time control of weld width in GMAW-P (Ref. 23). Shi et al. set up a visual sensing system for acquiring and processing the images of the weld pool and realized the control of weld pool width (Ref. 24). Zhang et al. reconstructed the weld pool surface based on captured laser reflection on an imaging plane, and the results showed that the method was capable of reconstructing weld pool surface with acceptable accuracy in GMAW-P (Refs. 25, 26). Almost all the visual sensing methods of weld pool in GMAW-P were during the base current period to detour the intense arc during peak current period. However, it is the heat input and arc force during the peak current periods that determine the weld shaping, especially the joint penetration depth. The oscillation frequency, which works well to characterize the joint penetration in GTAW, is not applicable in GMAW mainly due to complexity caused by the metal transfer.

From the analysis above, the present work aims at finding characteristic signals that are capable of characterizing joint penetration during the peak current period in GMAW$\mathrm{P}$ by visual sensing or other sensing technologies other than oscillation frequency of the weld pool. Wang et al. studied the relationship between a change in weld pool surface depth and a change in arc voltage, and proposed that the change in arc voltage during the peak current period can indeed provide an accurate prediction for the joint penetration depth during GMAW-P (Ref. 27). The change in weld pool surface depth and the change in arc voltage could be recognized as weld pool (surface) fluctuation amplitude, which would be determined by the weld pool size under the stimulation of pulse and droplet transfer in GMAW-P. When welding a material with a fixed thickness, the bigger the weld

Table 1-Parameters of the Laser

Model Wavelength/nm Power/mW

Fan angle/ deg
Laser

Pattern
Intensity Distribution 


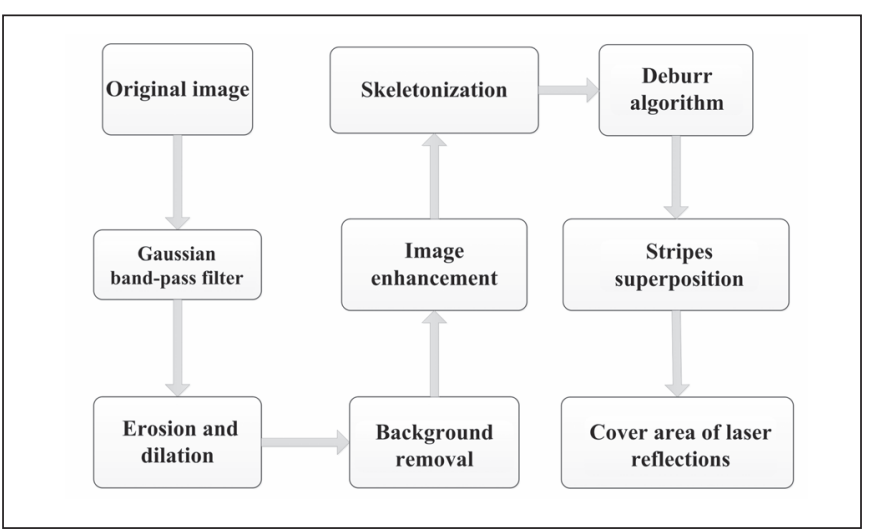

Fig. 3 - Flow chart for the image processing algorithm.

pool is, the smaller the fluctuation amplitude is due to the larger inertia of weld pool. Thus, the present work proposes the weld pool surface fluctuations during the peak current period as the target signal to characterize joint penetration.

\section{Experimental Design}

To express the weld pool surface fluctuations, visual sensing is an apparent solution. However, the bright arc during the peak current period becomes a huge challenge to obtain the visual information. Referring to the structured light reflection sensing method (Refs. 25, 26), weld pool surface information during the peak current period could be obtained. The main improvements described as following were done to try to get the clear laser reflection patterns: 1 ) a laser with raised power, $500 \mathrm{~W}$, was employed to enhance the pattern quality; 2) the wavelength of laser, $675 \mathrm{~nm}$, was chosen by arc spectrum, which showed the arc intensity is weak between the wavelength of 620 and $730 \mathrm{~nm}$; 3) a band filter with the wavelength $675 \pm 5 \mathrm{~nm}$ was accordingly used to further eliminate the arc; 4) a higher frame rate of camera was employed to try capturing the instantaneous fluctuations with high speed; 5) a larger imaging plane with a dimension of $260 \times 185 \mathrm{~mm}$ was used to collect the laser reflection pattern in a larger scale due to the severe fluctuations in GMAW-P. The joint penetration sensing system was established, as shown in Fig. 1. The laser, whose detailed parameters are listed in Table 1, projected nine lines onto the weld pool surface. The high-speed digital camera system, Photron FASTCAM Super $10 \mathrm{KC}$ with a shot speed of 30 to 10,000 frames/s, was used to capture images of laser reflection pattern on the imaging plane, and the images were synchronized with welding current and arc voltage signals (the two electrical signals will be discussed later). The fluctuations of laser reflection patterns obtained during the peak current period will be related to joint penetration to verify its effectiveness.

Another expression of weld pool fluctuations is arc voltage, because arc length will be varied with the fluctuations of weld pool, and arc length will affect the arc voltage. Among joint penetration sensing methods, the electrical signal sensing is one of the most ideal ones because 1 ) the welding current and arc voltage determine the heat input and arc force in the welding process, further determining
Table 2 - Welding Parameters of GMAW-P

\begin{tabular}{lc} 
Welding Parameters & Values \\
\hline Peak current (A) & 280 \\
Peak current period (ms) & 50 \\
Base current (A) & 50 \\
Pulse frequency (Hz) & 6 \\
Travel speed (mm/s) & 2.85 \\
Contact tip to workpiece & 15 \\
distance (mm) & \\
Gas flow rate (L/min) & 20 \\
\hline
\end{tabular}

the weld shaping; and 2) electrical signal acquisition and processing are simple and fast. Although the noises of electrical signals are usually large, they are still attractive and important signals to characterize the welding process. To eliminate the influence of welding current on arc voltage during the peak current period, the peak current and its duration were set as constant in the experiments. The variation amplitude of arc voltage and the average arc voltage during the peak current period will be analyzed and discussed for joint penetration control.

A single variable, wire feed speed (WFS), was used to obtain different joint penetrations. Other constant welding parameters are listed in Table 2 . The influence of metal deposition "WFS/TS" (TS is short for travel speed) on joint penetration was ignored in the present work; thus, WFS should be limited to a certain range (Ref. 27). WFSs were set at 3.3, $3.5,3.8,3.9,4.0,4.1,4.2,4.3,4.4,4.5,4.7$, and $4.8 \mathrm{~m} / \mathrm{min}$. In the experiments, the Fronius TPS- 4000 was used as a welding power supply with the wire feeder VR400. The base material used was Q235 steel plate of $270 \times 50 \times 4 \mathrm{~mm}$, and the filler wire was MG50-6 with a diameter of $1 \mathrm{~mm}$. The chemical compositions of base material and filler wire are shown in Table 3. Butt joint welding was conducted where the "I" groove was employed, and the opening between the two workpieces was $1 \mathrm{~mm}$.

\section{Experimental Results and Discussion}

\section{Visual Sensing Analysis of Different Weld Penetrations}

As shown in Fig. 1, a nine-line laser pattern was projected onto the weld pool surface, and the laser reflection patterns were captured by the high-speed camera from the imaging plane. The shot frequency was adjusted up to 7000 frames/s with the minimum exposure of camera. (It reached the limit of camera to get clear images before the droplet ran into the weld pool. If the shot frequency was more than 7000 frames/s, the exposure should be increased.) The laser reflection patterns still became chaos after the droplets ran into the weld pool, which are similar to images 32-36 in Fig. 2. Thus, another approach to get weld pool fluctuation was proposed.

The shot frequency was set constant at 3000 frames/s. A series of laser reflection patterns were obtained from one pulse, and some of them are shown in Fig. 2. During the 

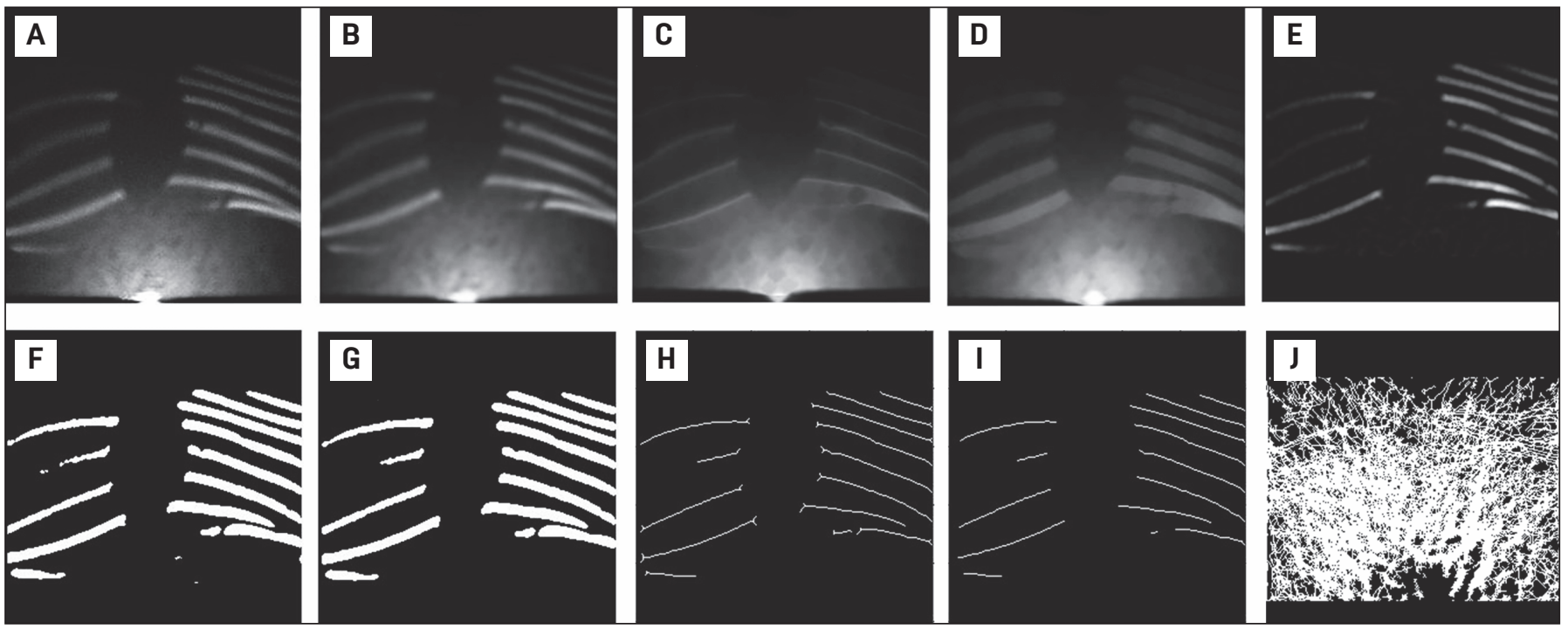

Fig. 4 - Image processing for laser refection pattern extraction: A - Original image; B - Gaussian band-pass filter; $C$ - erosion; $D$ - dilation; $E$ - background removal; $F$ - enhancement; $G$ - median filter; $H$ - skeletonization; I - deburr; I - superposition of all processed laser reflection patterns during a peak current period.

base current period (images 1-18), laser reflection patterns were relatively regular and fluctuated in a small range on the imaging plane, which were related to the stable weld pool surface owing to the minor base welding current. The positions of reflected laser patterns mostly distributed at the top of the images. When the projection angles of the projected laser lines are fixed, the smaller $\beta_{\mathrm{i}}$ is $\left(\beta_{\mathrm{i}}\right.$ is the inclination angle between the weld pool surface front and the workpiece and is the acute angle here), the higher the positions of reflected laser lines on the imaging plane are, according to the law of reflection. $\beta_{\mathrm{i}}$ is small during the base current period, thus the positions of reflected patterns at the top of the images. The weld pool is relatively stable during the base current period, since there is usually no droplet transferring into the weld pool during it, and the projected lasers are dense and uniform, therefore the laser concentrated on the imaging plane during the base current periods. The discontinuity in the middle of the stripes was caused by the occlusion of wire or droplets, which can also be observed in other conditions. Before droplet transfer during the peak current period (images 19-26), the stripes in the image transformed from aggregation state to divergent state and the spacing of stripes gradually became larger. This is because the depression on the weld pool surface increases with the increase in current.

The center part of the stripes produced depressions because of the weld pool surface depression caused by arc force. After droplet transfer during the peak current period (images 27-36), the stripes crossed and overlapped each other. The stripes became more chaotic with the increase in time. This is because the fluctuation of the weld pool surface spreads quickly to the other areas of weld pool surface when the droplet enters into the pool, and there was not enough time for the weld pool surface to return to a stable state as more droplets ran into the weld pool sequentially, so that the stripes chaos remained during the peak current period. From Fig. 2, the laser reflection patterns were regular during the base current period and early in the peak current period, but it became irregular later in the peak current period due to the droplet transfer. Thus, it is difficult to get accurate weld pool fluctuations during peak current period under the present conditions. However, the weld pool fluctuations during the peak current period might be estimated from the images by statistical approaches. Herein, the cover area of laser reflections $\left(A_{c}\right)$, which indicates the weld pool fluctuation amplitude, was proposed to characterize the joint penetration.

\section{Image Processing of Laser Reflection Patterns}

An image processing algorithm was proposed to extract the laser reflection pattern, whose flow chart is shown in Fig. 3 . The first objective is to segment the laser reflection pattern from the background arc light. Then the extracted pattern is linearized to remove the influence of the stripe width. Finally, the superposition of all processed laser reflection patterns during a peak current period was conducted to obtain $A_{c}$. An original image captured before droplet

Table 3 - Chemical Compositions of the Base Material and Filler Wire (wt-\%)

\begin{tabular}{cccccccc} 
Elements & $\mathrm{C}$ & $\mathrm{Mn}$ & $\mathrm{P}$ & $\mathrm{S}$ & $\mathrm{Si}$ & $\mathrm{Cu}$ & $\mathrm{Fe}$ \\
\hline Q235 & 0.18 & 0.50 & $\leqq 0.45$ & $\leqq 0.45$ & $\leqq 0.30$ & - & Balance \\
MG50-6 & 0.07 & 1.27 & 0.015 & 0.014 & 0.76 & 0.15
\end{tabular}


A

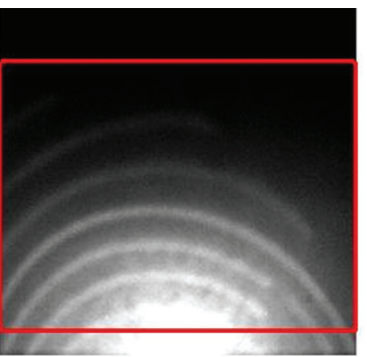

B

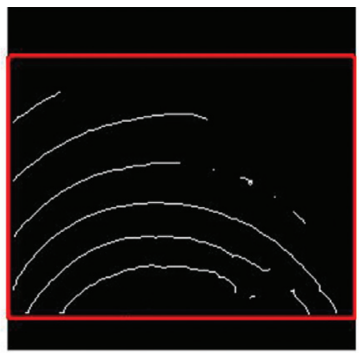

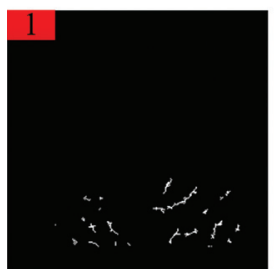
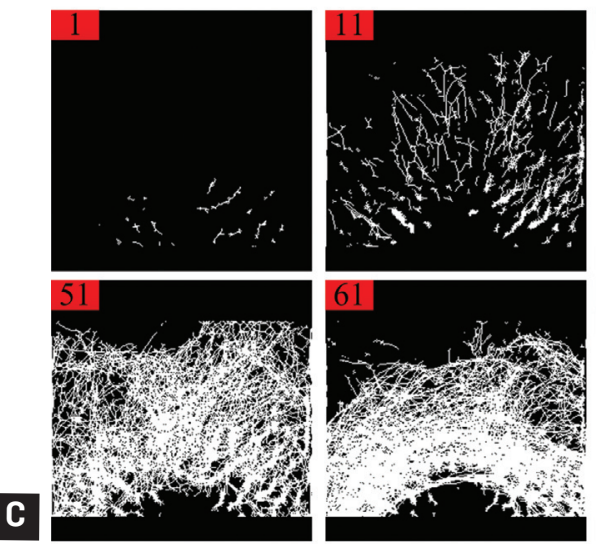
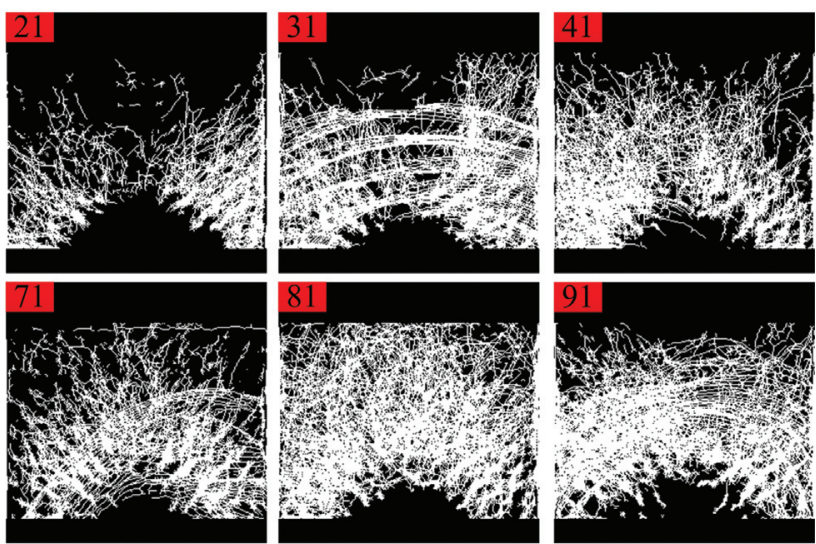

Fig. 5 - Image processing for cover area of laser reflections: $A$ - Original image and fixed window; $B$ - extracted laser reflection patterns within the fixed window; $C-A_{c}$ during a peak current period. The numbers on the top left corner of the images represent the sequence number of the pulse peak period.

transfer during a peak current period is shown in Fig. 4A. A filtered image, which used the Gaussian band-pass filter (described in Equations 1 and 2) to smooth the image and remove noise, was obtained, as shown in Fig. 4B

$$
\begin{aligned}
& H(x, y)=1-\exp \left(-\left(\frac{D(x, y)^{2}-D_{0}^{2}}{D(x, y) \cdot D_{1}}\right)^{2}\right) \\
& D(x, y)=\sqrt{\left(x-m_{1}\right)^{2}+\left(y-n_{1}\right)^{2}}
\end{aligned}
$$

where $D_{0}, D_{1}$ are band-pass cut-off frequencies, which are set to 125 and 580, $m_{1}$ and $n_{1}$ are all set to $128, H(x, y)$ represents the transfer function of the filter, and $D(x, y)$ is the distance from point $(x, y)$ to the filter center.

The morphological image processings of erosion and dilation were applied to the filtered image and the results are shown in Fig. 4C and D. The pattern became wider and more blurred since the erosion algorithm is used for finding the local grayscale minimum, and the dilation algorithm is used for finding local grayscale maximum. Image subtraction operation between Fig. 4B and D was applied to remove arc light background, and the result is shown in Fig. 4E. Figure $4 \mathrm{~F}$ is the result of the thresholding algorithm applied to Fig. 4E. From Fig. 4F, there were still some noises in the background. A median filter algorithm was then used to eliminate the noises in the background, whose result is shown in Fig. 4G. The morphological image processings of skele- tonization and deburr were applied to Fig. 4G, and the results are shown in Fig. $4 \mathrm{H}$ and I. The stripes location information could be obtained from Fig. 4I. It is difficult to obtain every fluctuation of each stripe from Fig. 4, but $A_{c}$, which presents the largest fluctuation area or fluctuation amplitude, could be easily obtained. Figure 4J superimposed images of extracted laser reflection pattern like Fig. 4I during a peak current period and then binarized the superimposed image. $A_{c}$ could thus be calculated by the sum of pixels of binarized superimposition of extracted laser refection patterns.

A fixed window was selected to eliminate the influence of image noises, as shown in Fig. 5A. Figure 5B shows the extracted laser reflection patterns within the fixed window. Figure $5 C$ shows the results of $A_{c}$ during different peak current periods. From Fig. $5 C, A_{c}$ during a peak current period varied in different pulse current cycles, which was related to the fluctuation amplitudes of the weld pool surface during different peak current periods.

\section{Visual Characteristic Signal Analysis for Joint Penetration Sensing}

The laser reflection patterns are closely related to the weld pool surface, and the laser reflection pattern will fluctuate with the weld pool surface, thus $A_{c}$ in the fixed window can reflect the amplitude of weld pool surface fluctuations to a certain degree. The information from the peak current period is stated to be closely related to the joint penetration, 

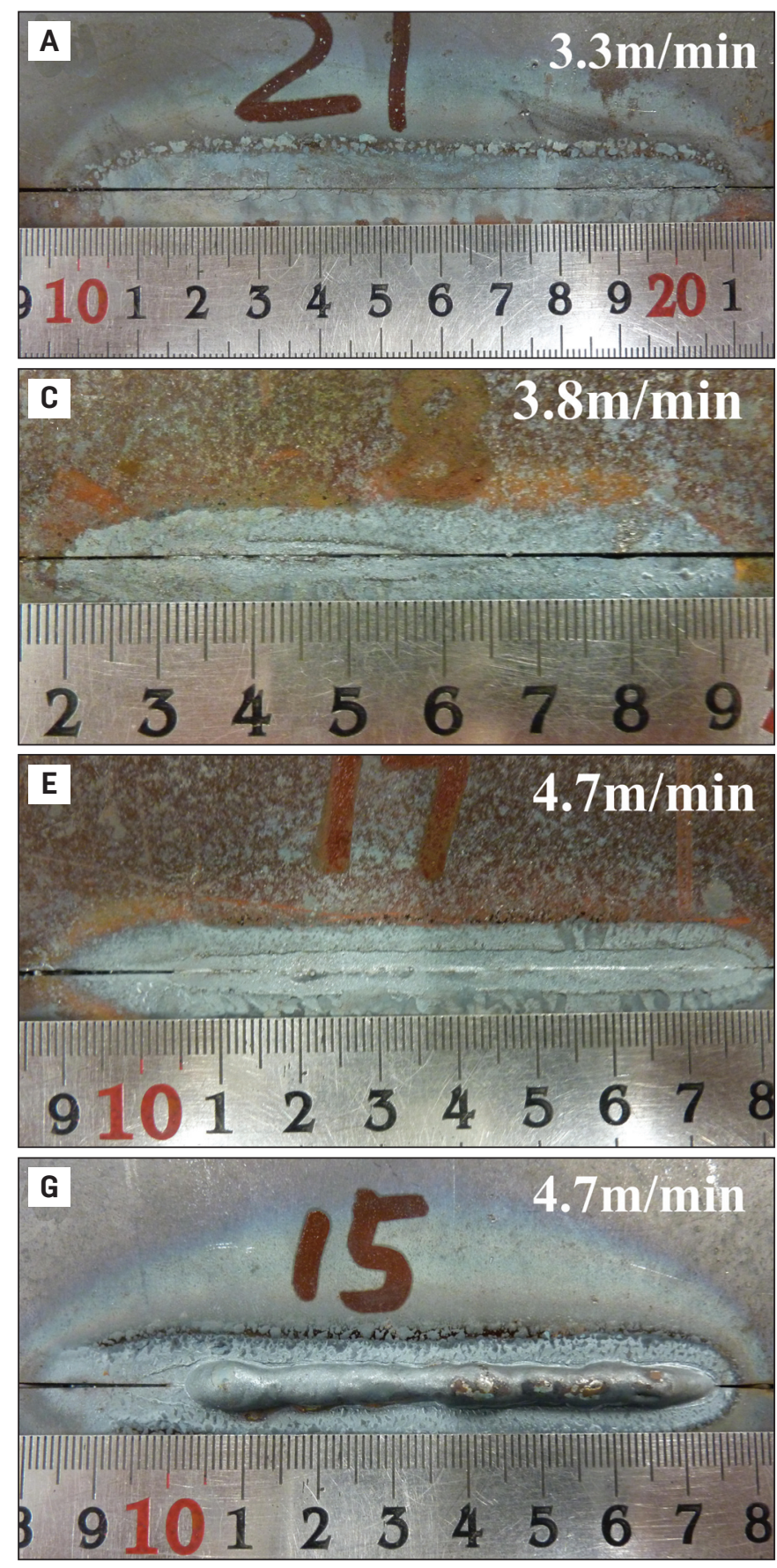
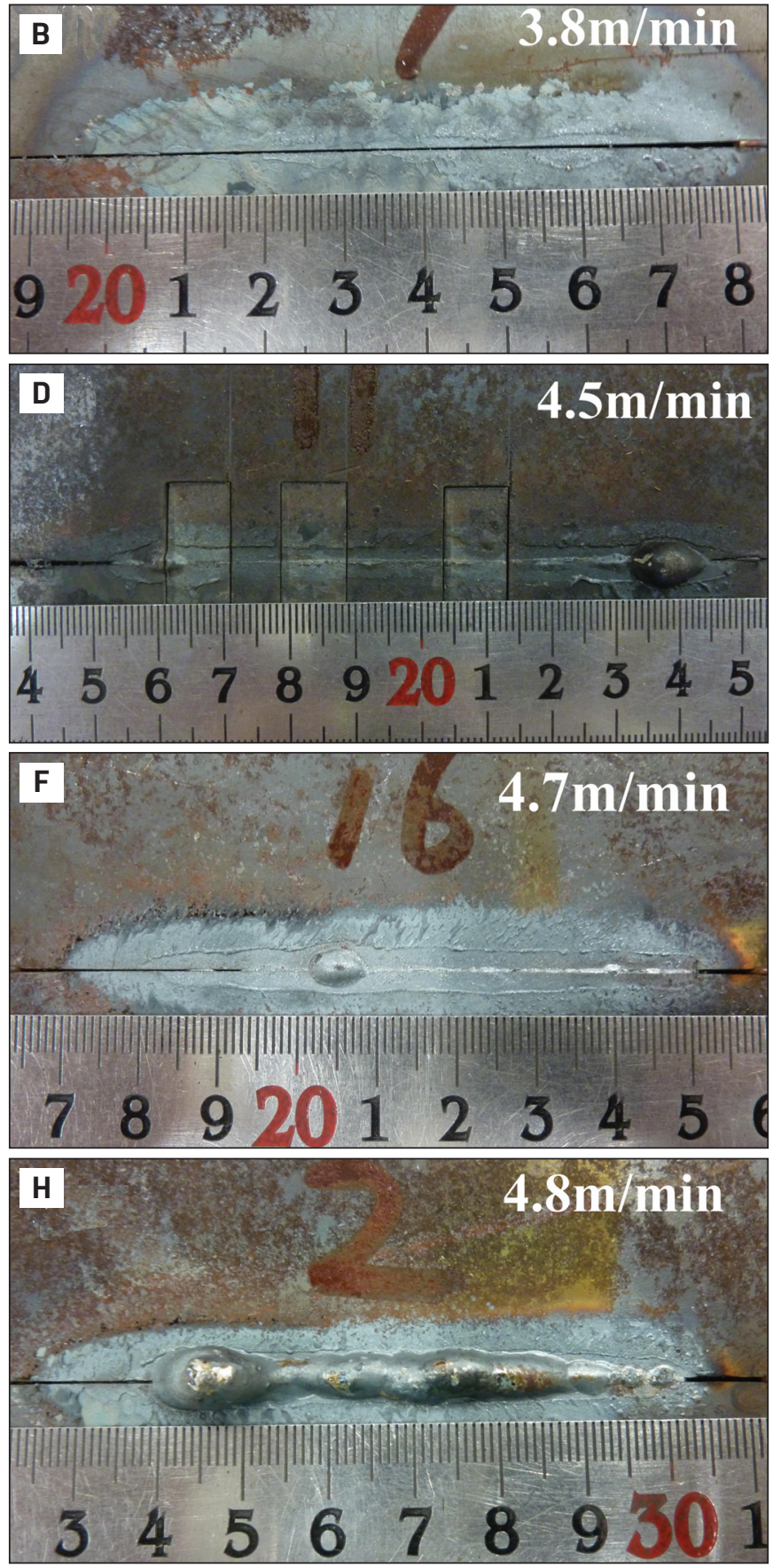

Fig. 6 - Backside weld appearance - A, B, and C are partial joint penetration welds at different WFSs; D, E, and F are critical penetration welds at different WFSs; $G$ and $H$ are complete joint penetration welds at different WFSs.

as mentioned above; therefore, $A_{c}$ during a peak current period was analyzed and discussed in detail.

A series of welds with different joint penetrations were obtained by changing WFS, whose backside appearances are shown in Fig. 6. Partial joint penetration welds in Fig. 6A-C were obtained at the WFS of 3.3 and $3.8 \mathrm{~m} / \mathrm{min}$, as marked in the figures. Additionally, the critical penetration welds in Fig. 6D-F were obtained at the WFS of 4.5 and $4.7 \mathrm{~m} / \mathrm{min}$, as marked. The complete joint penetration welds (shown in Fig. 6G and H) were obtained at the WFS of 4.7 and 4.8 $\mathrm{m} / \mathrm{min}$, as marked. The average cover area of laser reflec- tions in the stabilization stage of the welding process was obtained for different joint penetrations, as shown in Fig. 7. The alphabet marked in Fig. 7 corresponds to that in Fig. 6. From Figs. 6 and 7, $A_{c}$ during a peak current period for critical penetration welds was the highest, and that for complete joint penetration welds was the lowest. From partial to critical penetration, with the joint penetration depth increases, the volume of weld pool increases and the oscillation amplitude of weld pool surface decreases due to the increased inertia of the weld pool. Thus, the laser reflection pattern would appear in the fixed window with a higher probability, 


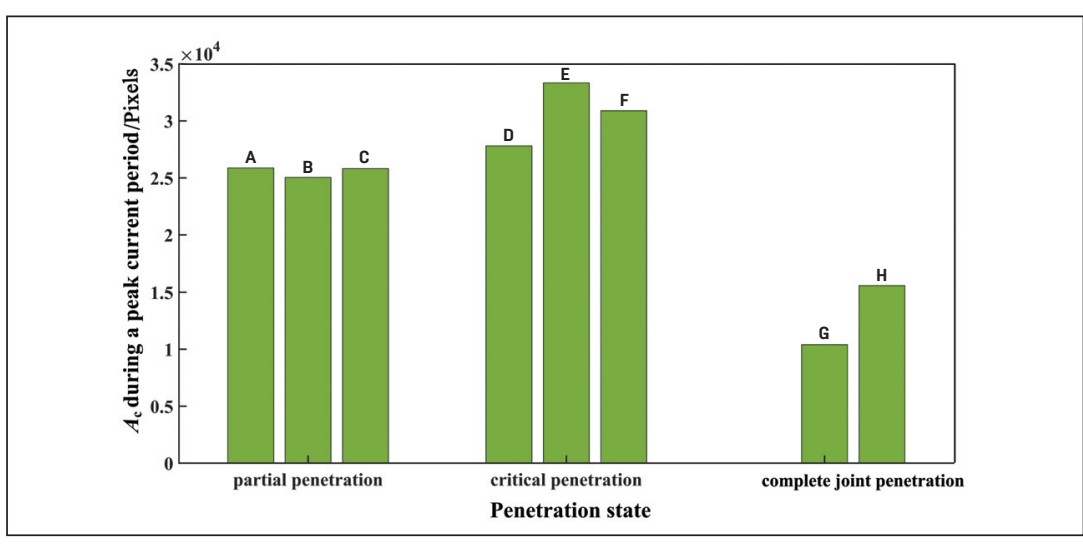

Fig. 7- The average $\mathrm{A}_{c}$ during a peak current period for different joint penetration states.
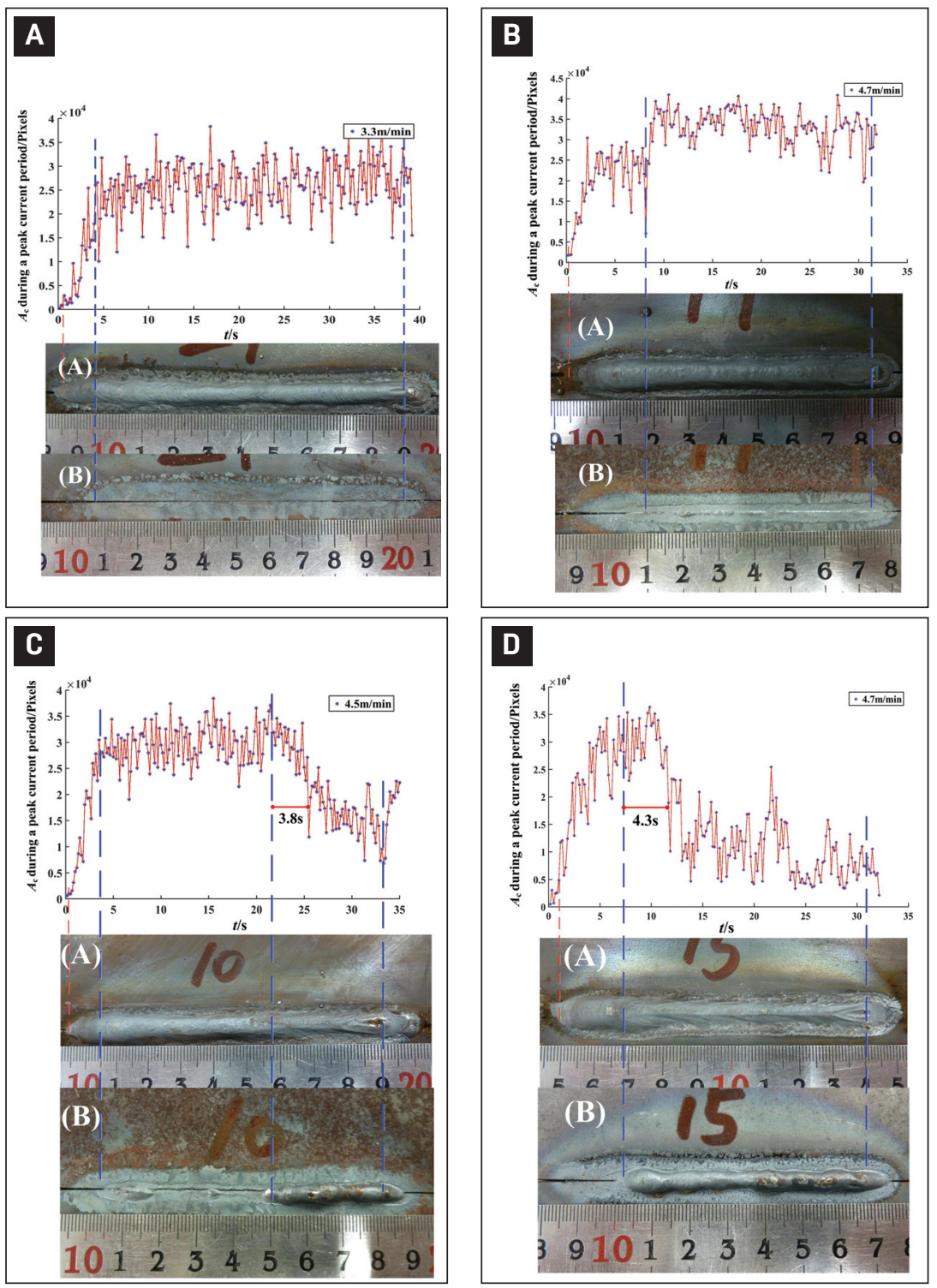

Fig. 8 - Relationship between joint penetration states and $\mathrm{A}_{\mathrm{c}}$ during a peak current period: $A-$ Partial joint penetration weld; $B$ - critical penetration weld; $C$ mix penetration weld from partial to complete joint penetration; $D$-complete joint penetration weld: $(A)$ the topside of the weld; $(B)$ the backside of the weld. namely that $A_{c}$ during a peak current period in the fixed window will increase if the joint penetration depth increases before critical penetration. From critical to complete joint penetration, the melted metal flows downward and weld pool fluctuation amplitude increases due to the absence of the backside constraint, thus the laser reflection will fall out of the fixed window, making $A_{c}$ during a peak current period decrease. From the analysis above, when the weld experiences the transition from partial to critical and then to complete joint penetration, $A_{c}$ during a peak current period tends to increase firstly and then to decrease.

A series of $A_{c}$ during peak current periods in different welding processes were obtained in real time, as shown in Fig. 8, in which the topside and backside welds were also presented. Therefore, the relationship between joint penetration states and $A_{c}$ can be analyzed and interpreted in real time. For partial joint penetration (Fig. 8A), the weld pool grew up at the beginning of welding, the fluctuation amplitude decreased, and $A_{c}$ during a peak current period in the fixed window increased. When the weld pool came into a relative stable state, $A_{c}$ fluctuated near a stable value. Figure $8 \mathrm{~B}$ shows a weld experienced the state from partial joint penetration to critical penetration. $A_{c}$ during a peak current period increased when its states changed from partial to critical penetration. Figure $8 \mathrm{C}$ shows a weld with the penetration state changed from partial to complete joint penetration (as called mix penetration, some parts of welds are in partial joint penetration and some parts of them are in complete joint penetration). When complete joint penetration was achieved, the weld pool had a depression from the view of the topside weld, which had a direct influence on fluctuation amplitude and further made $A_{c}$ during a peak current period decrease. Also, the depression had an influence on arc voltage, which will be discussed in the next section. For complete joint penetration (Fig. 8D), $A_{c}$ during a peak current period was at a relatively low level, around $1.5 \times 10^{4}$ pixels. The experimental results from Fig. 8 show $A_{c}$ during a peak current period in the fixed window can dynamically reflect the fluctuation amplitude of the weld pool surface and further be used for real-time sensing of joint penetration, but with a time delay - Fig. 8C and D. From Figs. 7 and $8, A_{c}$ during a peak current period will have a sudden drop when the weld gets penetrated. The complete joint penetration state is identified when $\mathrm{A}_{c}$ falls lower than $1.5 \times 10^{4}$ pixels for the first time from a high value. But the position of identified turning point from partial to complete joint penetration falls behind the visible turning point at the backside of the weld, so apparent time delay ex- 


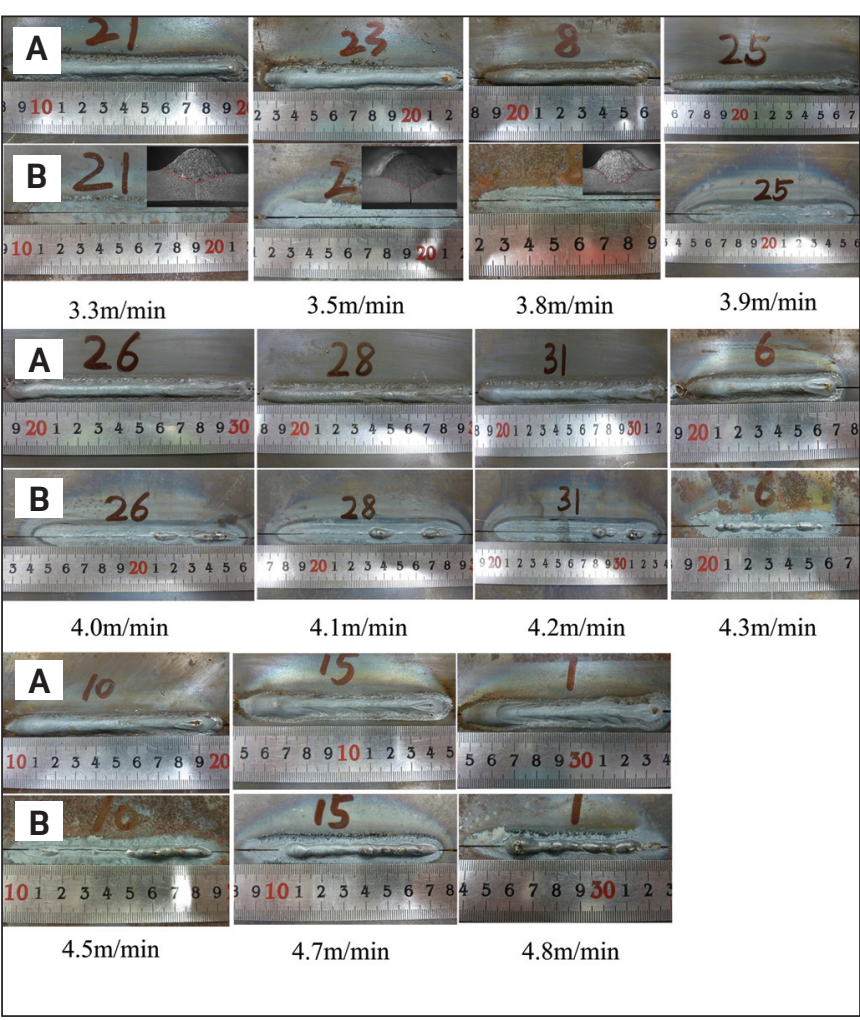

Fig. 9 - The topside (A) and backside (B) of weld appearance at different WFSs.

ists in the system, which is expressed by the time interval between the two turning points. In Fig. $8 C, A_{c}$ during a peak current period decreased to a lower level after a time delay of $3.8 \mathrm{~s}$ when the joint penetration state changed from partial to complete joint penetration. In Fig. 8D, the time delay was $4.3 \mathrm{~s}$ after the weld got penetrated.

\section{Electrical Signal Sensing Analysis of Different Joint Penetrations}

As previously mentioned, the depression of the weld pool surface has an influence on arc length further on arc voltage. Thus, arc voltage during a peak current period will be discussed in detail. Weld pool fluctuation amplitude has an influence on arc length variation, too, and the change in arc voltage, during a peak current period (Refs. 27, 28), will also be analyzed.

Figure 9 shows the weld appearances from the top and bottom side, in which the joint penetration gradually changed from partial to complete joint penetration with the increase in WFS. Welding current and arc voltage signals were acquired for all the experiments in Fig. 9. Bai et al. (Ref. 29) reported that the average change in arc voltage during a peak current period $(\Delta U)$ could predict the joint penetration at the beginning of the GMAW-P process. When the welding process came into stable state, Wang et al. (Refs. 27,28 ) and Bai et al. (Ref. 29) both used $\Delta U$ to characterize the joint penetration depth before critical penetration in GMAW-P. The present work extends the aforementioned research to complete joint penetration in GMAW-P. A series of

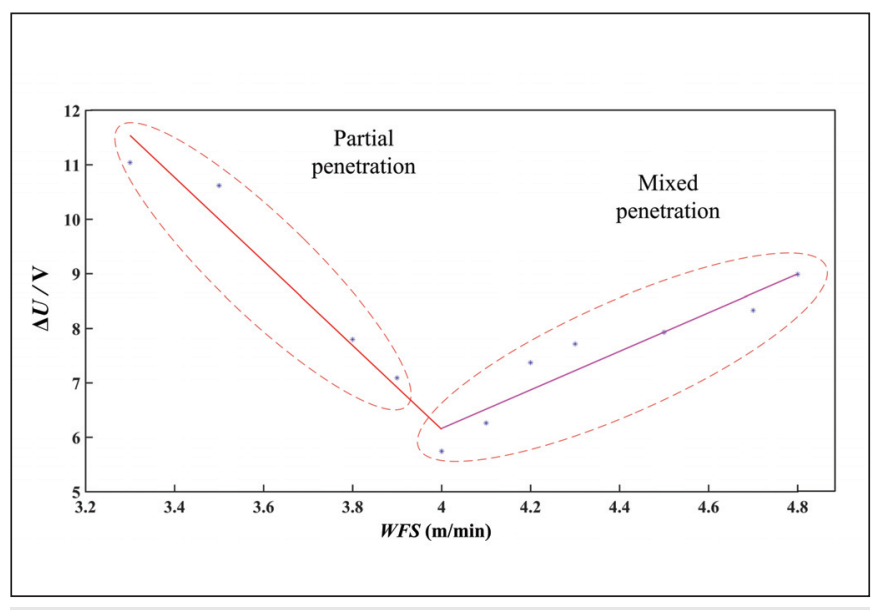

Fig. 10 - The relationship between $\Delta \mathrm{U}$ and WFS.

$\Delta U$ in stable state of welding processes at different WFSs were obtained, as shown in Fig. 10.

As shown in Figs. 9 and 10, with the increase in WFS, $\Delta U$ decreased firstly and then increased after WFS was over $4.0 \mathrm{~m} / \mathrm{min}$. For WFS below $4.0 \mathrm{~m} / \mathrm{min}$, the welds were almost in partial joint penetration (Fig. 9). When WFS was over $4.0 \mathrm{~m} / \mathrm{min}$, the welds were in mixed penetration, or almost in complete joint penetration (Fig. 9). When WFS is below $4.0 \mathrm{~m} / \mathrm{min}$, with the increase in WFS, the heat efficiency of welding processes will increase, the joint penetration depth (and the size of weld pool) will increase, and the increase in mass and further in the inertia will decrease the fluctuation amplitude of weld pool, which will cause the decrease in fluctuation amplitude of arc length and further that of the arc voltage. When it comes into the critical penetration, $\Delta U$ comes into the minimum. When the workpiece is penetrated (WFS is over $4.0 \mathrm{~m} / \mathrm{min}$ ), the molten metal in the weld pool will flow downward under the arc force and the gravity, and fluctuation amplitude of the weld pool surface will increase owing to the absence of constraint; further $\Delta U$ will increase again.

Complete joint penetration gradually dominated in the whole weld with the increase in WFS from 4.0 to 4.8 $\mathrm{m} / \mathrm{min}$ (Fig. 9), causing the increase in $\Delta U$ (Fig. 10). Two welds of different penetrations matching with the $\Delta U-t$ chart are shown in Fig. 11. $\Delta U$ was filtered by the simple moving average (SMA) with different sample widths ( $n$ ) (Ref. 30), which means a moving window of $n$ samplings of $\Delta U$ is defined firstly and then the mean of the values in the window is calculated to obtain a series of filtered $\Delta U$ by moving this window along the original data. From Fig. 11, $\Delta U$ for critical penetration was at a lower level compared to partial and complete joint penetration. The positions between the partial and complete joint penetration or those with a narrow backside width among complete joint penetration positions, which are marked by red lines in Fig. 11, were considered as in critical penetration. The waveforms of $\Delta U$ near the positions in critical penetration have a similar contour, namely that there is a valley right there or a time lagged behind. It confirmed the results from Fig. 10 that $\Delta U$ decreases firstly and then increases when the weld is from partial to complete joint penetration. The wave- 

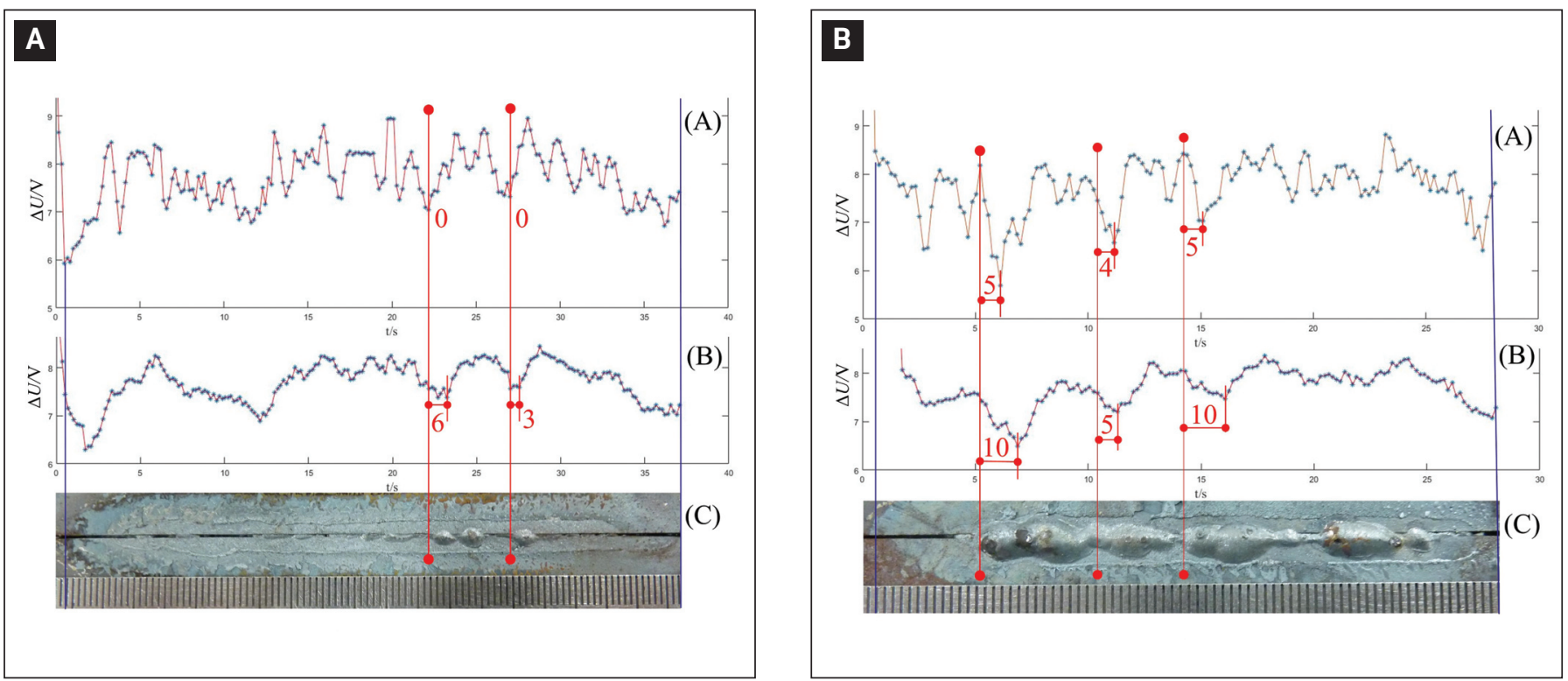

Fig. 11 - Relationship between $\Delta \mathrm{U}$ and joint penetration: $11 \mathrm{~A}$ and $11 \mathrm{~B}$ were two joint penetration states at the WFS of $4.3 \mathrm{~m} / \mathrm{min}$; (A) $-\Delta \mathrm{U}-\mathrm{t}$ when $\mathrm{n}$ is equal to 3 ; $(\mathrm{B})-\Delta \mathrm{U}-\mathrm{t}$ when $\mathrm{n}$ is equal to $10 ;(\mathrm{C})$ - backside weld morphology. The number next to the vertical line in the figure represents the delayed periods of $\Delta \mathrm{U}$ sampling.

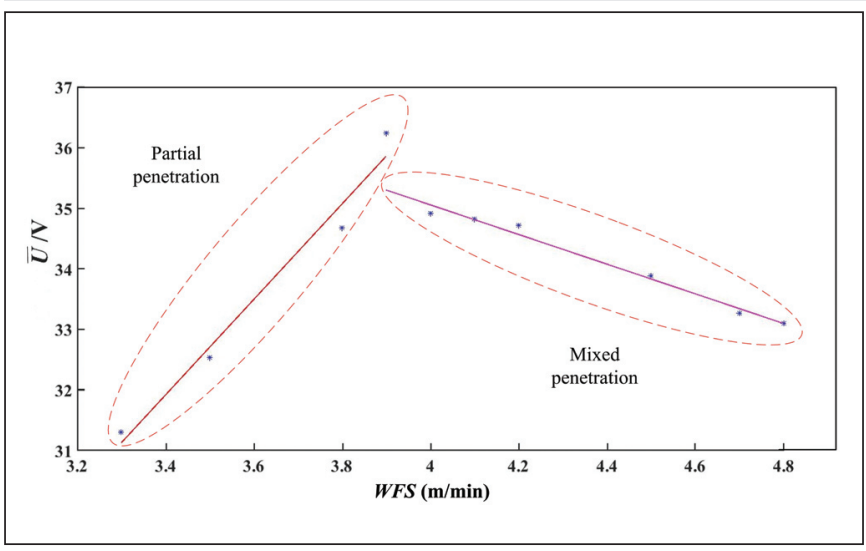

Fig. 12 - The relationship between $\overline{\mathrm{U}}$ and WFS.

forms for the critical penetration position among complete joint penetration positions can also be explained by Fig. 10. From Fig. 11, $\Delta U$ lagged behind the change of joint penetration, and the time delay of Group A was less than that of Group B. The time delay was caused by the inertia thermal welding process and SMA algorithm used. The difference in the time delay mainly stemmed from the SMA algorithm. The greater $n$ was set to be, the more high-frequency signal filtered from $\Delta U$ but the greater the time delay was. When $n$ was too low, the $\Delta U$ signal was interfered by noises and it became difficult to tell joint penetration from $\Delta U$, although the delay of $\Delta U$ decreased. A suitable value of $n$ should be selected when designing the control strategies of $\Delta U$ in joint penetration control.

As mentioned above, the weld pool had a depression from the view of the topside weld when complete joint penetration was achieved. The depression is closely related to arc length and further the arc voltage, thus average arc voltage during a peak current period $(\bar{U})$ was considered to charac- terize joint penetration. The $\bar{U}$ is defined in Equation 3 ,

$$
\bar{U}=\left(\sum_{i=1}^{n} \bar{U}_{i}\right) / n
$$

where $\bar{U}_{i}$ is the average arc voltage during a peak current period, and $\bar{U}$ is an average of $\bar{U}_{i}$ and the default $n=10$. The relationship between $\bar{U}_{i}$ and WFS was obtained, as shown in Fig. 12.

From Figs. 12 and 9, for partial joint penetration, $\bar{U}$ increased with the increase in WFS; and for the mixed penetration state, $\bar{U}$ decreased with the increase in WFS. This phenomenon could be explained by the principle of minimum voltage. With the increase in WFS, the arc length will be kept in a new equilibrium point, where the energy for melting the wire meets the wire feed speed. Due to the peak current and peak current period were set as constant in the present work, the arc will change its shape to make the arc voltage drop for unit length minimum. In such case, 1) if the weld is in partial joint penetration, the arc length will become shorter (although the weld pool surface will have the trend to lengthen arc length by deformation, the solid constraint from the backside of the workpiece and the larger variation of WFS make the arc length shorter), but the electric field strength will be much larger, resulting in a raising $\bar{U}$; 2) if the complete joint penetration was achieved, the absence of solid constraint from the backside of the workpiece would make the electrical field strength decrease and make the arc length trend to increase suddenly, but it was counteracted by the arc shortening due to the increase in WFS, thus $\bar{U}$ will decrease after critical penetration.

The $\bar{U}$ representing the depression of the weld pool, and $\Delta U$ representing the fluctuation amplitude of the weld pool, are both important control variables in joint penetration control. These two variables could be fused together to control joint penetration to solve the problem that the electrical 
signals in welding processes are easily affected by the noises. They are easily measurable and applicable in joint penetration control in GMAW-P in manufacturing plants.

\section{Conclusions}

This paper studied the characteristic information of joint penetration by monitoring the weld pool surface fluctuation with the methods of structured laser reflection and electrical signal sensing in pulsed gas metal arc welding (GMAW$\mathrm{P})$. The cover area of laser reflections $\left(A_{c}\right)$ during a peak current period, the change in arc voltage during a peak current period $(\Delta U)$, as well as the average voltage during a peak current period $(\bar{U})$ in different joint penetrations were analyzed. Several conclusions can be drawn:

1) Fluctuation amplitude of the weld pool surface, which is determined by the weld pool size under the stimulation of pulse and droplet transfer in GMAW-P, can be used to characterize the joint penetration. The deeper the joint penetration is and the bigger the weld pool is, the smaller the fluctuation amplitude of the weld pool surface is, due to the larger inertia of the weld pool.

2) The $A_{c}$ during a peak current period can reflect the fluctuation amplitude of the weld pool surface and further can be used to characterize the joint penetration. The relationship between $A_{c}$ during a peak current period and joint penetration was analyzed, and the results showed that $A_{c}$ during a peak current period for critical penetration welds was the highest, and that for complete joint penetration welds was the lowest, making that $A_{c}$ during a peak current period can be used for recognition and control of joint penetration. It provided a new idea for the vision sensing and control of the joint penetration.

3) The $\Delta U$ is an easily measurable variable to express the weld pool surface fluctuation. The present work extended $\Delta U$ from partial to complete joint penetration. From partial to complete joint penetration, $\Delta U$ decreases firstly then increases. $\bar{U}$ representing the depression of weld pool was also analyzed. From partial to complete joint penetration, $\bar{U}$ increases firstly then decreases. These two variables could be fused together to control joint penetration in GMAW-P in manufacturing plants.

\section{Acknowledgments}

This research is supported by the National Natural Science Foundation of China (Grant No.: 51505326), Natural Science Foundation of Tianjin (Grant No.: 16JCQNJC04300), and the Regional Demonstration Project of Marine Economic Innovation and Development (Grant No. BHSF2017-10). We would like to thank Dr. Li Huan for the high-speed camera.

\section{References}

1. Zhao, D. B., Yi, J. Q., Chen, S. B., et al. 2004. Surface shape reconstruction of weld pool during pulsed GTAW from its single image.
Robotic Welding, Intelligence and Automation. Springer, Berlin, Heidelberg: 56-62. DOI: https://doi.org/10. 1007/9783-1540-44415-2_4

2. Wang, J. F., Wang, W. Y., and Chen, S. B. 2009. Inspection of welding pool height from shading in pulsed GTAW with wire filler. Industrial Robot 36(3): 270-276. DOI: doi.org/10.1108/ 01439910910950540

3. Kovacevic, R., and Zhang, Y. M. 1996. Sensing free surface of arc weld pool using specular reflection: Principle and analysis. Proceedings of the Institution of Mechanical Engineers, Part B - Journal of Engineering Manufacture 210(62): 553-564. DOI:

doi.org/10.1243/PIME_PROC_1996_210_154_02

4. Song, H., and Zhang, Y. M. 2007. Image processing for measurement of three-dimensional GTA weld pool surface. Welding Journal 86(10): 323-s to 330-s.

5. Song, H. S., and Zhang, Y. M. 2007. Three-dimensional reconstruction of specular surface for a gas tungsten arc weld pool. Measurement Science \& Technology 18(12): 3751-3767. DOI: 10.1088/0957-0233/18/12/010/pdf

6. Zhang, W. J., Liu, Y. K., and Zhang, Y. M. 2013. Real-time measurement of the weld pool surface in GTAW process. IEEE International Conference on Imaging Systems and Techniques 80(11): 1640-1645. DOI: dx.doi.org/10.5772/53753

7. Wu, C. S., and Gao, J. Q. 2006. Vision-based neuro-fuzzy control of weld penetration in gas tungsten arc welding of thin sheets. International Journal of Modelling Identification \& Control 1(2): 357-367. DOI: doi.org/10.1504/IJMIC.2006.010090

8. Zhang, Y. M., Kovacevic, R., and Li, L. 1996. Characterization and real-time measurement of geometrical appearance of the weld pool. International Journal of Machine Tools \& Manufacture 36(7): 799-816. DOI: 10.1016/0890-6955(95)00083-6

9. Bicknell, A., Smith, J. S., and Lucas, J. 1994. Arc voltage sensor for monitoring of penetration in TIG welds. IEE Proceedings Science Measurement and Technology 141(6): 513-520. DOI: 10.1049/ip-smt:19941144

10. Xiao, Y. H., and den Ouden, G. 1993. Weld pool oscillation during GTA welding of mild steel. Welding Journal 72(8): 428-s to 434-s.

11. Aendenroomer, A. J. R., and den Ouden, G. 1998. Weld pool oscillation as a tool for penetration sensing during pulsed GTA welding. Welding Journal 77(5): 181-s to 187-s.

12. Wang, Q. L., Yang, C. L., and Geng, Z. 1993. Separately excited resonance phenomenon of the weld pool and its application. Welding Journal 72(9): 455-s to 462-s.

13. Shi, Y., Zhang, G., Ma, X. J., et al. 2015. Laser-vision-based measurement and analysis of weld pool oscillation frequency in GTAW-P. Welding Journal 94(5): 176-s to 187-s.

14. Chen, J. S., Chen, J., Zhang, K., et al. 2018. Dynamic reflection behaviors of weld pool surface in pulsed GTAW. Welding Journal 97(6): 191-s to 206-s.

15. Zhang, K., Zhang, Y. M., Chen, J. S., et al. 2017. Observation and analysis of three-dimensional weld pool oscillation dynamic behaviors. Welding Journal 96(5): 143-s to 153-s.

16. Hardt, D. E., and Katz, J. M. 1984. Ultrasonic measurement of weld penetration. Welding Journal 63(9): 273-s to 281-s.

17. Guu, A. C., and Rokhlin, S. I. 1989. Computerized radiographic weld penetration control with feedback on weld pool depression. Materials Evaluation 47(10): 1204-1210.

18. Rokhlin, S. I., and Guu, A. C. 1990. Computerized radiographic sensing and control of an arc welding process. Welding Journal 69(3): 83-s to 97-s.

19. Beardsley, H. E., Zhang, Y. M., and Kovacevic, R. 1994. Infrared sensing of full penetration state in gas tungsten arc welding. International Journal of Machine Tools \& Manufacture 34(8): 1079-1090. DOI: doi.org/10.1016/0890-6955(94)90014-0

20. Wang, H., and Kovacevic, R. 2002. Feasibility study of acoustic sensing for the welding pool mode in variable-polarity plasma arc welding. Proceeding of the Institute of Mechanical Engi- 
neers, Part B - Journal of Engineering Manufacture 216(10): 1355-1366.

21. Yan, Z., Wu, L., Zhang, G., et al. 2004. Visual sensing and profile extraction of the weld pool in pulsed gas metal arc welding. Proceeding of the Institute of Mechanical Engineers, Part B - Journal of Engineering Manufacture 218(10): 1333-1338. DOI: doi.org/10.1243/0954405042323441

22. Zhang, G., Yan, Z., and Wu, L. 2006. Reconstructing a threedimensional P-GMAW weld pool shape from a two-dimensional visual image. Measurement Science \& Technology 17(7): 1877-1882. DOI: 10.1088/0957-0233/17/7/028/pdf

23. Chen, S. B., Cao, J. M., Xu, C. M., et al. 2002. Visual sensing and real-time control of weld pool dynamics in pulsed GMAW. Transactions of the China Welding Institution 23(4): 17-20 (in Chinese).

24. Shi, Y., Fan, D., and Huang, A. 2005. Vision-based identification model of welding pool width dynamic respondence in aluminum alloy pulsed MIG process. Acta Metallrugica Sinica 41(9): 994-998.

25. Wang, Z. Z., Zhang, Y. M., and Ma, X. J. 2011. Machine recognition of laser reflection from gas metal arc weld pool surfaces. Journal of Manufacturing Science \& Engineering 133(4):
041013. DOI: $10.1115 / 1.4004498$

26. Ma, X. J., and Zhang, Y. M. 2013. Reconstruction of threedimensional gas metal arc weld pool surface from reflected laser pattern. Journal of Manufacturing Science \& Engineering 135(2): 021002. DOI: 10.1115/1.4023374

27. Wang, Z., Zhang, Y. M., and Wu, L. 2010. Measurement and estimation of weld pool surface depth and weld penetration in pulsed gas metal arc welding. Welding Journal 89(6): 117-s to 126-s.

28. Wang, Z., Zhang, Y. M., and Wu, L. 2012. Adaptive interval model control of weld pool surface in pulsed gas metal arc welding. Automatica 48(1): 233-238. DOI: 10.1016/ j.automatica.2011.09.052

29. Bai, P., Wang, Z., Hu, S., et al. 2017. Sensing of the weld penetration at the beginning of pulsed gas metal arc welding. Journal of Manufacturing Processes 28: 343-350. DOI:

10.1016/j.jmapro.2017.07.002

30. Johnston, F. R., Boyland, J. E., Meadows, M., et al. 1999. Some properties of a simple moving average when applied to forecasting a time series. Journal of the Operational Research Society 50(12): 1267-1271. DOI: doi.org/10.1057/

palgrave.jors. 2600823

\section{Authors: Submit Research Papers Online}

Peer review of research papers is now managed through an online system using Editorial Manager software. Papers can be submitted into the system directly from the Welding Journal page on the AWS website (aws.org) by clicking on "submit papers." You can also access the new site directly at editorialmanager.com/wj/. Follow the instructions to register or log in. This online system streamlines the review process, and makes it easier to submit papers and track their progress. By publishing in the Welding Journal, more than 70,000 members will receive the results of your research.

Additionally, your full paper is posted on the American Welding Society website for FREE access around the globe. There are no page charges, and articles are published in full color. By far, the most people, at the least cost, will recognize your research when you publish in the world-respected Welding Journal. 